\title{
Salmonella Infection in Mesenteric Lymph Nodes of Breeding Sows
}

\author{
Victoria Garrido,, Samanta Sánchez,, Beatriz San Román,, Lorenzo Fraile, \\ Lourdes Migura-García, ${ }^{3}$ and María-Jesús Grilló ${ }^{1}$
}

\begin{abstract}
Salmonellosis is one of the main foodborne diseases worldwide. Breeding sows asymptomatically infected with Salmonella can transmit the pathogen to piglets and humans. The isolation of Salmonella from mesenteric lymph nodes (MLNs) is considered a demonstration of asymptomatic infection in swine. As previous breeding sow studies have been performed using feces, the aim of this work was to study the occurrence of Salmonella infections by sampling MLNs, in comparison to their serological status. First, Salmonella fecal shedding was studied in 12/16 large breeding farms to establish the framework of study. Then, MLN $(n=264)$ and blood $(n=237)$ samples were obtained at an abattoir from sows of 15 of these 16 breeding farms. Additionally, risk factors associated with Salmonella MLN infection were analyzed. A total of 6.1\% (16/264) sows, distributed in $40 \%(6 / 15)$ of the farms, had the pathogen in MLN. Salmonella Typhimurium was the most frequent serovar isolated. Interestingly, $43.8 \%$ (7/16) of MLN isolates were susceptible to all the antimicrobials tested and were found distributed throughout all farms with at least one sow positive. As well, one isolate of the emerging DT195 clone was detected and found to be resistant to six antibiotic families (ASSuTNx-Cfx). The serovars and the resistance profiles of the Salmonella isolates from feces were completely different to those obtained from MLNs. The seroprevalence ( $41.8 \%$ of sows and $100 \%$ of farms) was higher than that of MLN infections, showing no concordance $(k=0.15)$ between these two diagnostic tests in sows. Strategies directed to correct two risk factors (i.e., administration of dry food and old premises) would most likely help to reduce Salmonella infections in breeding sows.
\end{abstract}

Keywords: Salmonella, prevalence, sows, lymph nodes, shedding, antimicrobial resistance, risk factors

\section{Introduction}

$\mathbf{N}$ ONTYPHOIDAL SALMONELLOSIS is a worldwide-distributed zoonosis caused by Salmonella, a pathogen of public health relevance. After initial control in fowl, pig products are emerging as an important source of Salmonella for humans in the European Union (EU) (EFSA-ECDC, 2015; EFSA-ECDC, 2018). To protect the health of the consumers, the EU is legislating for the reduction of Salmonella in pigs and derivate food, including breeding sows as a vertical source of infection of the pathogen to the litter (DOUE, 2003).

Spain is the fourth largest country in pig production, after China, United States, and Germany, showing an increase of $228.5 \%$ sow offal (i.e., entrails and organs used for food processing) export from 2008 to 2017 (MAPA, 2018). Results of the salmonellosis EU baseline studies indicated that
Spain was on the top of Salmonella prevalence at pig herd level, detecting the bacteria in pooled fecal samples (PFS) from $64 \%$ of breeding holdings (EFSA, 2008, 2009). Salmonella can be present in feces after an active infection of enterocytes and lymphatic system and/or by passive transmission of the bacteria through the gut after ingestion. Sows infected asymptomatically can shed the pathogen intermittently contaminating the environment (Scherer et al., 2008) and transmitting it to humans and piglets (Casanova-Higes et al., 2019). Reliable diagnostics of Salmonella infection can only be assessed by the pathogen isolation in the gut wall or lymphatic system, being mesenteric lymph nodes (MLNs) the sample of choice (DOUE, 2003; EFSA, 2006). However, no information about Salmonella prevalence in sow MLN is available, probably owing to practical limitations for sampling sows in the abattoir. In some EU countries, serological

\footnotetext{
${ }^{1}$ Department of Animal Health, Instituto de Agrobiotecnología (CSIC-Gobierno de Navarra), Mutilva, Spain.

${ }^{2}$ Department of Animal Health, Universidad de Lleida, Lleida, Spain.

${ }^{3}$ Department of Animal Health, Institut de Recerca i Tecnologia Agroalimentàries (IRTA), Centre de Recerca en Sanitat Animal (CReSA), Barcelona, Spain.
} 
diagnosis is considered an alternative in Salmonella control programs in fattening pigs (Mousing et al., 1997; Merle et al., 2011; Meroc et al., 2012). Nevertheless, no correlation was observed between serology and the presence of Salmonella in MLN or fecal samples of these animals (San Román et al., 2018).

The objectives of this study were to determine: (1) prevalence and type of Salmonella present in sow MLN; (2) seroprevalence and the usefulness of the enzyme-linked immunosorbent assay (ELISA) as a tool to assess Salmonella MLN infection in sows, at individual and at herd levels; and (3) main risk factors associated to MLN infection.

\section{Materials and Methods}

\section{Sampling design}

The research was performed in Navarra, a region in Northern Spain with moderate-low prevalence of Salmonella in fattening pigs (San Román et al., 2018). In this context, a total of 65,308 breeding sows kept on 763 farms were registered in 2014 (INTIA, personal communication). Most of these animals (37,964 sows) were kept on only 16 large farms, which contained more than 1200 sows each and were managed by 7 integrator companies. These large herds were based on a closed replacement system using gilts born in the farm, with a replacement rate of around $50 \%$.

To know the framework of the research, we determined the prevalence of Salmonella shedding at herd level, in comparison to that observed in the EU baseline study (EFSA, 2009). For this, individual fecal samples were obtained in 12 out of the 16 large farms of Navarra and processed in pools of 5 ( $25 \mathrm{~g} / \mathrm{PFS})$, as previously described (San Román et al., 2018). The fecal samples were obtained at farm proportionally to: (1) the number of rooms of each reproductive unit (i.e., gestation, farrow, replacement, and confirmed gestation); and (2) the number of sows kept in each room, representing the different reproductive cycle stages. Accordingly, 340 sows housed in gestation units and 205 sows housed in farrowing units were sampled in 12 farms, however, only 30 sows housed in replacement units in 4/12 farms and 25 sows housed in confirmed gestation units in $3 / 12$ farms could be analyzed. Thus, a total of 600 individual fecal samples from 5 or 10 rooms/farm were collected from the rectum by using the double-glove method and individual sterile containers to avoid cross-contamination.

Within this framework, 15 out of the 16 large farms containing a total of 33,545 sows were included in the MLN infection and serological study. The sows sampled were selected from those regularly sent by the farmer to the abattoir for breeding replacement, thus, all sows were multiparity, but the parity number was unknown. Owing to the lack of previous information on Salmonella MLN infection in sows, 15-20 animals/farm (depending on the herd size) were considered representative to detect the presence of Salmonella infection, assuming an estimated minimum prevalence of $20 \%$ by farm and a $95 \%$ confidence interval $\left(\mathrm{CI}_{95 \%}\right)$. The pooling was not considered for sample size calculations, since pooling was highly efficient compared with individual sampling in previous studies (Arnold et al., 2009). Accordingly, a total of 264 sows were sampled for MLN and 237 of them (one farm failed) were also bled at the abattoir and used as paired samples for concordance studies.

\section{Salmonella isolation and characterization}

Both MLN $(n=264)$ and fecal $(n=120)$ samples were processed by the ISO 6579:2002/Amd 1:2007 (hereafter, ISO 6579) (ISO, 2007), as previously detailed (San Román et al., 2018). Presumptive Salmonella isolates were sent to the National Center for Animal Salmonellosis (Algete, Madrid, Spain) for confirmation and serotyping by the KauffmanWhite Scheme (Grimont and Weill, 2007). Thereafter, Salmonella Typhimurium isolates were phage typed by standardized protocols in the National Center of Microbiology at the Instituto de Salud Carlos III (Madrid, Spain) according to standard protocols (Echeíta et al., 2005).

All Salmonella isolates were analyzed by the KirbyBauer disk diffusion test (CLSI, 2013) in cation-adjusted Mueller-Hinton plates against 12 antimicrobials (all from BD, Spain) of 7 different families, as previously detailed (Garrido et al., 2014). The antimicrobials tested were: ampicillin and amoxicillin/clavulanic acid (aminopenicillins; A); chloramphenicol (phenicols; C); streptomycin and gentamicin (aminoglycosides; S); sulfisoxazole, trimethoprim, and trimethoprim/sulfamethoxazole (sulfonamides; Su); tetracycline (tetracyclines; T); nalidixic acid (quinolones; $\mathrm{Nx}$ ); ciprofloxacin (fluoroquinolones; Cip); and cefotaxime (third-generation cephalosporins; Cfx). Escherichia coli ATCC 25922, Salmonella Typhimurium ATCC 14028 and DT104 were used as controls in each experiment. Clinical breakpoints to classify isolates as susceptible or resistant were defined by the Clinical and Laboratory Standards Institute (CLSI, 2013). Isolates were considered multidrug resistant when exhibiting resistance to at least three antimicrobial families.

\section{Serological study}

Blood samples $(n=237)$ were obtained at abattoir, individual sera were extracted by centrifugation $\left(4^{\circ} \mathrm{C}, 10 \mathrm{~min}\right.$, $1500 \times g$ ) in a Multifuge 3L-R (Sorvall, Heraeus), and analyzed by Herd-Check ${ }^{\circledR}$ Swine Salmonella indirect ELISA test (IDEXX $^{\mathrm{TM}}$ Laboratories, Switzerland). This test had shown an optimal sensitivity and specificity in fattening pigs $(88 \%$ and $74 \%$, respectively) compared with other commercial kits (Vico et al., 2010). Optical density (O.D.) values were normalized and analyzed at different cutoff values (i.e., 10\%, $20 \%$, and $40 \%$ ), according to the manufacturer's instructions.

\section{Concordance test}

Considering the $100 \%$ specificity of bacteriology, a farm was considered positive when Salmonella was confirmed in at least one MLN sample. Concordance analysis between infection and serology was performed by Kappa test $(k)$ using MLN and blood paired samples $(n=237)$. Descriptive statistics and prevalence were estimated with a $\mathrm{CI}_{95 \%}$. Statistical comparison of percentages was performed by a Chisquare test with Fisher's correction $(p \leq 0.05)$ when required, using the SPSS 15.0.1 statistical software (SPSS, Inc., Chicago, IL).

\section{Questionnaire data and statistical analysis}

A questionnaire consisting of 70 variables was used to assess possible risk factors associated to Salmonella MLN infection. Questions were divided into five main sections in 
the farm survey: (1) farm general characteristics related to herd size, number of gestation units, and number of full-time workers; (2) biosecurity aspects such as existence and maintenance of outside fence and footbath, use of specific clothes, entrance restrictions, rodent control programs, and presence of cats, dogs, and wild birds; (3) feeding: type of feed, number of diets, and water supplier; (4) use of antimicrobial agents: type, number and length of treatments; and (5) farmeŕs personal information: age, educational level, and additional training on pig production. To provide reliable information, all the surveys were checked for reliability and filled out with the assistance of the farm veterinarian.

For statistical analysis, an initial risk factors' screening was carried out by a univariable Chi-square test. The variables detected as significant $(p \leq 0.05)$ were further considered in a multivariable random-effect logistic regression model in which the outcome variable was the "culture positive"; the explanatory variables included in the model as fixed effect were those from the questionnaire; and the random effect was the farm. Multivariable analysis was performed by the STATA software (StataCorp, L.P., College Station, TX). An odds ratio $(\mathrm{OR})>1$ indicated that animal exposure to the factor increases the risk of Salmonella positivity, whereas an OR $<1$ indicates a reduced risk of animal positivity due to exposure to the factor.

\section{Results}

\section{Salmonella prevalence at farm level in fecal samples}

The framework of the study was defined by the presence of Salmonella in 13/120 (10.8\%) PFS, which were from 50\% $(6 / 12)$ of farms studied (Table 1). Farms with at least one positive PFS showed a $21.6 \%$ mean prevalence, but $58.3 \%$ of these farms exhibited low levels of contamination, since they had $\leq 10 \%$ positive PFS (Table 1 ). As shown in Table 2, farms from integrators $\mathrm{B}$ and $\mathrm{F}$ showed a higher proportion of positive PFS. A wide variety of serovars, but not Typhimurium, were identified in these samples (Table 2). All the isolates were pansusceptible $(6 / 11)$ or only resistant to streptomycin $(5 / 11)$ alone or combined with tetracycline (Table 2).

\section{Salmonella MLN infection in sows}

As shown in Table 1, Salmonella spp. was found in 16/264 (6.1\%) MLN of sows, which were in 6/15 (40\%) breeding farms. Within positive farms, the mean prevalence of the pathogen was $14.5 \%$, but $80 \%$ of the farms showed $\leq 10 \%$ animals infected, displaying a marked left-biased distribution of the infection (Fig. 1).

A total of 6 serovars from 4 different serogroups were detected in sows' MLN (Table 2). The most common serovar was Typhimurium (43.7\%) followed by Derby (18.7\%), Enteritidis (12.5\%) and Montevideo (12.5\%) (Table 2). A total of $43.7 \%(7 / 16)$ of MLN isolates were pansusceptible and found distributed in all positive farms except one (i.e., Farm 4) (Table 2). The remaining 56.3\% (9/16) MLN isolates (serovars Typhimurium or Derby) showed multiresistance to three (SSuT) or more (ACSSuT and ASSuT-Nx-Cfx) antimicrobial families. These three different multidrug-resistance patterns were found in three different farms (Table 2). The SSuT Derby isolates were common to Farms 2 and 4, and the two different multidrug-resistant Typhimurium were restricted to one origin each (i.e., Farms 1 or 4). In fact, the five Typhimurium isolates found in Farm 1 showed the typical pentaresistant profile (ACSSuT) associated to DT104 phage type, and the DT195 found in Farm 4 showed a particular ASSuT$\mathrm{Nx}-\mathrm{Cfx}$ multidrug-resistant profile. Overall, the distribution of the Salmonella phenotypes suggested a different origin of infection for each farm.

\section{Seroprevalence}

All farms studied had at least one seropositive sow, even at the maximum $40 \%$ O.D. ELISA cutoff. The mean seroprevalence was $41.8 \%$ (Table 1) varying from $15 \%$ to $80 \%$ between farms (Table 2), but all farms showed $>10 \%$ seroprevalence (Fig. 1). These results indicated that seroprevalence was higher than MLN infection. The discrepancy between both diagnostic techniques was evidenced by (1) the 8 farms that showed $25-80 \%$ of seropositive sows but none of them infected in MLN (Table 2); and (2) the high proportion (86/99) of sows negative by bacteriology but positive in ELISA (Table 3). Accordingly, no concordance between serology and microbiology was statistically confirmed by a $k=0.15$ Kappa index.

\section{Risk factor analysis of Salmonella $M L N$ infection in sows}

The risk factor questionnaire was accurately completed by $13 / 15$ of the farms and two farms were excluded because their

Table 1. Prevalence of Salmonella in Mesenteric Lymph Nodes and Serology (Enzyme-Linked Immunosorbent Assay) of Sows Sampled at Abattoir and in Pooled Fecal Samples Obtained at the Farms Where the Sows Were Kept

\begin{tabular}{|c|c|c|c|}
\hline & $\begin{array}{c}M L N \\
\left(\text { mean } \% ; C I_{95 \%}\right)\end{array}$ & $\begin{array}{c}\text { Serology } y^{\mathrm{a}} \\
\left(\text { mean \%; } C I_{95 \%}\right)\end{array}$ & $\begin{array}{c}P F S \\
\left(\text { mean } \% ; C I_{95 \%}\right)\end{array}$ \\
\hline No. of positive ${ }^{b} /$ total no. of samples & $16 / 264(6.1 ; 3.7-9.6)$ & $99 / 237(41.8 ; 35.7-48.1)$ & $13 / 120(10.8 ; 6.4-17.6)$ \\
\hline No. of positive ${ }^{b} /$ total no. of farms & $6 / 15(40 ; 19.8-64.2)$ & $14 / 14(100 ; 78.5-100)$ & $6 / 12(50.0 ; 25.3-74.6)$ \\
\hline $\begin{array}{l}\text { No. of positive pigs } / \text { total no. of pigs } \\
\text { analyzed in positive farms }\end{array}$ & $16 / 110(14.5 ; 9.1-22.3)$ & $99 / 237(41.8 ; 35.7-48.1)$ & $13 / 60(21.6 ; 13.1-33.6)$ \\
\hline $\begin{array}{l}\text { No. }(\%) \text { of farms with } \leq 10 \% \\
\text { prevalence/total no. of farms }\end{array}$ & $12 / 15(80.0)$ & $0 / 14(0)$ & $7 / 12(58.3)$ \\
\hline
\end{tabular}

a $40 \%$ O.D. cutoff.

${ }^{\mathrm{b}}$ Pigs or farms where at least 1 colony-forming unit of Salmonella spp. was isolated.

$\mathrm{CI}_{95 \%}, 95 \%$ confidence interval; MLN, mesenteric lymph node; O.D., optical density; PFS, pooled fecal samples. 


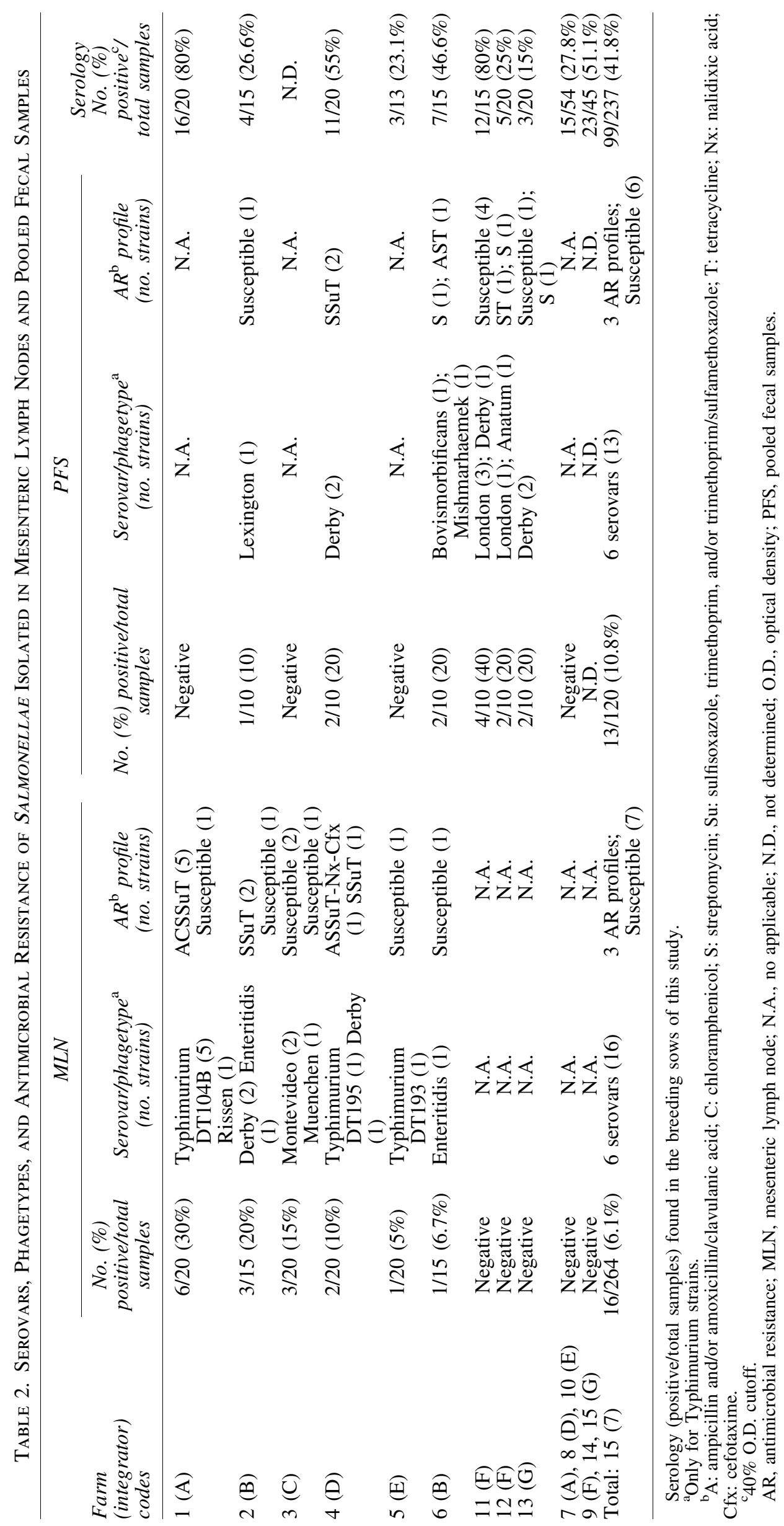




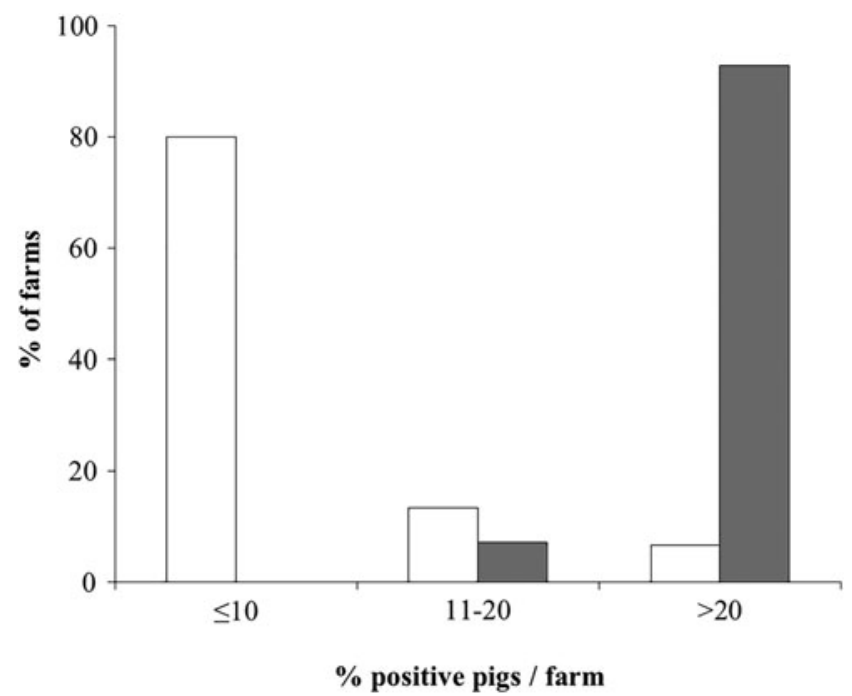

FIG. 1. Distribution of Salmonella in breeding sows, expressed as the percentage of farms (ordinates) showing $\leq 10 \%, 11-20 \%$, or $>20 \%$ individual prevalence (abscissas). MLN (white bars) and blood serum (grey bars) were analyzed by the standard ISO 6579 and by the IDEXX ${ }^{\circledR}$ ELISA test at $40 \%$ O.D. cutoff, respectively. MLN, mesenteric lymph node; O.D., optical density.

data were unconsistant. Since these two farms showed all sows free from Salmonella infection, the eventual risk factor analysis was carried out with data from 6 farms showing 5$30 \%$ of Salmonella-infected sows and 7 farms showing all sows free from Salmonella infection.

In the univariate scrutiny, a total of 12/70 variables were initially associated with Salmonella MLN infection. From them, only two variables remained significant in the subsequent multivariable logistic regression model (Table 4), indicating that (1) administration of dry food (as compared with food mixed with water); and (2) lack of shed/barn/building renovations in the last 5 years were significant risk factors.

\section{Discussion}

All previous studies of salmonellosis in sows have been performed with fecal samples. However, the presence of Salmonella in feces could be due not only to excretion from

Table 3. Distribution of the Serological and Microbiological Salmonellosis Results IN Paired Samples of Sows $(N=237)$

\begin{tabular}{lrcc}
\hline & \multicolumn{2}{c}{ MLN } & \\
\cline { 2 - 3 } No. of sows & Positive & Negative & Total samples \\
\hline Serology & & & \\
$\quad$ Positive & 13 & 86 & 99 \\
$\quad$ Negative & 0 & 138 & 138 \\
Total samples & 13 & 224 & 237 \\
\hline
\end{tabular}

Sera and MLN samples were obtained from each sow at abattoir, and analyzed by the IDEXX ${ }^{\circledR}$ ELISA test at $40 \%$ O.D. cutoff and the standard ISO 6579 method, respectively.

MLN, mesenteric lymph node; O.D., optical density.
Table 4. Epidemiological Variables Significantly Associated With Salmonella Prevalence IN MESENTERIC Lymph Node OF Sows

\begin{tabular}{lcc}
\hline & \multicolumn{2}{c}{ Logistic regression parameters } \\
\cline { 2 - 3 } Explanatory variable & $\mathrm{p}$ & OR $(95 \%$ CI $)$ \\
\hline $\begin{array}{l}\text { Food administration } \\
\text { Mixed with water }\end{array}$ & & 1 \\
$\begin{array}{l}\text { Dry } \\
\text { Shed/barn/building }\end{array}$ & 0.005 & $9(1.9-39.4)$ \\
$\quad$ renovations & & 1 \\
Yes $^{\mathrm{a}}$ & & \\
No & & \\
Constant & 0.042 & $3.01(1.04-8.73)$ \\
\hline
\end{tabular}

Determined by multivariable random effects logistic regression analysis after clustering pigs by farm of origin and considering MLN culture positive as the outcome variable.

${ }^{a}$ Reference category assigned as $\mathrm{OR}=1$ for statistical purposes.

CI, confidence interval; MLN, mesenteric lymph node; OR, odds ratio.

MLN but also to cross-contamination or passive ingestion and survival of the pathogen in the intestinal tract of the animal. Thus, Salmonella isolation in MLN is the most reliable way to demonstrate asymptomatic infection in swine (DOUE, 2006). Also, MLN infection is considered as a reservoir and a source of intermittent excretion and dissemination of the pathogen to piglets (EFSA, 2011). However, to our knowledge, only one previous study has been published about Salmonella MLN infection in breeding sows (Keteran et al., 1982). The prevalence found there $(58.2 \%)$ was higher than that found in our conditions $(6.1 \%)$, however, both studies are not exactly comparable, since animals of the former were maintained for $10 \mathrm{~d}$ in lairage, while in our study all sows were slaughtered within $2 \mathrm{~h}$ after arrival. Supporting our results, the level of infection found in sow MLN (6.1\%) was similar to that observed in fattening pigs MLN (7.2\%) of the same region (San Román et al., 2018).

Herein, we present a novel study of Salmonella infection in sows MLN and its concordance analysis with serology in 237 sows paired samples. Overall, moderate-low prevalence prevalence of Salmonella was observed in sows' MLN in the Navarra vertically integrated production system. This finding is in agreement with the moderate-low prevalence observed in fattening pigs in this production system of Navarra (San Román et al., 2018).

A large discrepancy between Salmonella infection and seropositivity $(41.7 \%)$ was observed. It was higher than the results previously reported for fattening pigs (San Román et al., 2018). Our observation of no concordance $(k=0.15)$ could be attributed to endemicity of infection in breeding holdings, higher possibility of reinfections, or antigenic contacts in breeding sows than in young pigs (Vico et al., 2010, 2011; Meroc et al., 2012). Additionally, a longer persistence of humoral immune response than infection itself could also be an explanation (Scherer et al., 2008). Other hypotheses such as lack of ISO 6579 sensitivity (MainarJaime et al., 2013), lack of ELISA specificity (Vico et al., 2011), or absence of exotic serogroup antigens in the ELISA plate coating (Van Winsen et al., 2001) could also contribute. However, serology has been applied in large studies for 
salmonellosis control in Denmark, Germany, and Belgium, where infection and serology are considered highly correlated in finishing pigs (Mousing et al., 1997; Merle et al., 2011; Meroc et al., 2012). Our results indicate that the serological diagnosis is of very limited efficacy (if any) to control salmonellosis in sows, at least in our epidemiological scenario.

In the EU baseline study, more than 50 different Salmonella serovars were found in feces of breeding sows, being Derby $(23.9 \%)$ and Typhimurium $(17.9 \%)$ the most frequent (EFSA, 2009). Contrarily, in our study, Salmonella Typhimurium was not detected in feces but was the serovar most frequently identified in MLN. Since this serovar is the most commonly reported in human infections in 2014 (EFSAECDC, 2016), future works could be focused on the study of sow offal (including internal organs and lymph nodes) as a possible source of human infections.

Besides the foodborne hazard, the inappropriate use of antimicrobial agents in humans (ECDC, 2018) and animals (EMA-ESVM, 2018) has led to a quick emergence of multidrug-resistant Salmonella of special epidemiological surveillance (DOUE, 2003). At the EU, 48.7\% of sow feces isolates were multidrug resistant (EFSA-ECDC, 2019), whereas only $18.2 \%$ were found in our framework. In contrast to PFS, we found high proportion (56.2\%) of multidrug-resistant Salmonella invading sow MLN, highlighting the phenotypic differences between both biological niches. Notably, one Salmonella Typhimurium DT195 was resistant to antimicrobials of six different families, including third-generation cephalosporins. This finding represents a proportion of cefotaxime-resistant isolates $(0.4 \%)$ lower to that observed in EU (1.2\%) and Spain (1.1\%) (EFSAECDC, 2019). This particular clone should be monitored to avoid the expansion of emerging genetic mobile elements carrying resistance to multiple antibiotics of interest in human treatments.

Previous information indicated that a main risk factor associated to Salmonella fecal shedding in sows was a high replacement rate by external gilts (Davies et al., 2000). In Navarra, the intensive production of breeding sows was based on a closed self-replacement with gilts born in the herd, which could contribute positively to the moderate-low prevalence observed in our study. In this study, the main risk factors associated to MLN infection were administration of dry food (instead of food mixed with water) and maintenance of sows in nonrenovated farms. The former could be associated to a lower persistence of Salmonella in feed water after acidic fermentation (Van Winsen et al., 2000; Missotten et al., 2015); and the latter, to an inefficient disinfection of old materials and/or the presence of Salmonella vectors, such as lizards, birds, or rodents (Andrés-Barranco et al., 2014). Besides strategies directed to correct these risk factors, other measures could be implemented to reduce MLN infections in sows, such as nutritional programs, including the addition of organic acids or prebiotics (Andrés-Barranco et al., 2015). Since some studies suggest a vertical dissemination of Salmonella from breeding to fattening pigs (EFSA, 2011), the application of all these control measures in sows would contribute to improve the control of this important zoonosis at farm level, to minimize the risk of pork food contamination from farm to fork and to preserve the competitiveness of this economical sector.

\section{Acknowledgments}

We are grateful to farmers, veterinaries, and slaughterhouse workers and to short-term students Naroa Remondegui, June Landa (JAE-Intro CSIC-FEDER fellowship), Ruth Erro and Mirian Samblas (FEUN-CSIC). We thank John Wild for the English revision.

\section{Disclosure Statement}

No competing financial interests exist.

\section{Funding Information}

The work was financed by Departamento de Industria, Energía e Innovación of the Navarra Government (reference IIQ14064. RI1) and INTIA-CSIC contract (reference CAM2011030054). V.G., S.S., B.S.R., and L.M.G's. contracts were funded by UPNA postdoctoral fellowship, EMUNDUS18 Program, CSIC JAEDoc Program, and INIA-European Social Fund, respectively.

\section{References}

Andrés-Barranco S, Vico JP, Garrido V, Samper S, HerreraLeón S, de Frutos C, Mainar-Jaime RC. Role of wild bird and rodents in the epidemiology of subclinical salmonellosis in finishing pigs. Foodborne Pathog Dis 2014;11:689-697.

Andrés-Barranco S, Vico JP, Grilló MJ, Mainar-Jaime RC. Reduction of subclinical Salmonella infection in fattening pigs after dietary supplementation with a $\beta$-galactomannan oligosaccharide. J Appl Microbiol 2015;118:284-294.

Arnold ME, Cook AJ. Estimation of sample sizes for pooled faecal sampling for detection of Salmonella in pigs. Epidemiol Infect 2009;137:1734-1741.

Casanova-Higes A, Marín-Alcalá CM, Andrés-Barranco S, Cebollada-Solanas A, Álvarez J, Mainar-Jaime RC. Weaned piglets: Another factor to be considered for the control of Salmonella infection in breeding pig farms. Vet Res 2019;50:45.

CLSI (Clinical and Laboratory Standards Institute). Performance Standards for Antimicrobial Disk and Dilution Susceptibility Tests for Bacteria Isolated From Animals; Approved Standard Fourth Edition. CLSI document VET01A4. Wayne, PA: CLSI, 2013.

Davies PR, Funk JA, Morgan-Morrow WE. Fecal shedding of Salmonella by gilts before and after introduction to a swine breeding farm. Swine Health Prod 2000;8:25-29.

DOUE Regulation (EC) No 2160/2003 of the European Parliament and of the council on the control of Salmonella and other species food-borne zoonotic agents. Official J Eur Union 2003;46:1-15. Available at: https://eur-lex.europa.eu/legalcontent/EN/TXT/?uri=OJ:L:2003:325:TOC, accessed November $27,2019$.

DOUE Commission Decision of 29 September 2006 concerning a financial contribution from the Community towards a baseline survey on the prevalence of Salmonella in slaughter pigs to be carried out in the Member States. 2006/668/EC. Official J Eur Union 2016;49:51-61. Available at: https://eurlex.europa.eu/legal-content/EN/TXT/?uri=uriserv:OJ.L_ 2006.275.01.0051.01.ENG\& toc=OJ:L:2006:275:FULL, accessed November 27, 2019.

ECDC (European Centre for Disease Prevention and Control). Antimicrobial consumption. In: ECDC. Annual Epidemiological Report for 2017. Stockholm: ECDC, 2018. Available at: https://www.ecdc.europa.eu/en/publications-data/antimicrobialconsumption-annual-epidemiological-report-2017, accessed November 27, 2019. 
Echeíta MA, Aladuena AM, Díez R, Arroyo M, Cerdán F, Gutierrez R, de la Fuente M, González-Sanz R, Herrera-Leon S, Usera MA. Serotype and phage type distribution of human Salmonella strains isolated in Spain, 1997-2001. Enferm Infecc Microbiol Clin 2005;23:127-134.

EFSA (European Food Safety Authority). Opinion of the scientific panel on biological hazards on Risk assessment and mitigation options of Salmonella in pig production. EFSA J 2006;341:1-131.

EFSA (European Food Safety Authority). Report of the Task Force on Zoonoses data collection on the analysis of the baseline survey on the prevalence of Salmonella in slaughter pigs. Part A. EFSA J 2008;135:1-111.

EFSA (European Food Safety Authority). Analysis of the baseline survey on the prevalence of Salmonella in holdings with breeding pigs in the EU, 2008. Part A: Salmonella prevalence estimates. EFSA J 2009;7:1377.

EFSA (European Food Safety Authority). Analysis of the baseline survey of Salmonella in holdings with breeding pigs, in the EU, 2008; Part B: Analysis of factors potentially associated with Salmonella pen positivity. EFSA J 2011;9:2329.

EFSA-ECDC (European Food Safety Authority and European Centre for Disease Prevention and Control). The European Union summary report on trends and sources of zoonoses, zoonotic agents and food-borne outbreaks in 2013. EFSA J 2015;13:3991.

EFSA-ECDC (European Food Safety Authority and European Centre for Disease Prevention and Control). The European Union summary report on trends and sources of zoonoses, zoonotic agents and food-borne outbreaks in 2015. EFSA J 2016;14:1-231.

EFSA-ECDC (European Food Safety Authority and European Centre for Disease Prevention and Control). The European Union summary report on trends and sources of zoonoses, zoonotic agents and food-borne outbreaks in 2017. EFSA J 2018;16:5500.

EFSA-ECDC (European Food Safety Authority and European Centre for Disease Prevention and Control). The European Union summary report on antimicrobial resistance in zoonotic and indicator bacteria from humans, animals and food in 2017. EFSA J 2019;17:5598.

EMA-ESVM (European Medicines Agency, and European Surveillance of Veterinary Antimicrobial Consumption). Sales of veterinary antimicrobial agents in 30 European countries in 2016. EMA/275982/2018. Available at: https://www.ema.europa .eu/en/veterinary-regulatory/overview/antimicrobial-resistance/ european-surveillance-veterinary-antimicrobial-consumptionesvac, accessed November 27, 2019.

Garrido V, Sánchez S, San Román B, Zabalza-Baranguá A, DíazTendero Y, de Frutos C, Mainar-Jaime RC, Grilló MJ. Simultaneous infections by different Salmonella strains in mesenteric lymph nodes of finishing pigs. BMC Vet Res 2014;10:59.

Grimont PA, Weill FX. Antigenic Formulae of the Salmonella Serovars. World Health Organization Collaboration Centre for Reference Research on Salmonella. Paris: Institute Pasteur, 2007.

International Organisation for Standardisation (ISO) 6579:2002/ Amd 1:2007. Microbiology of Food and Animal Feeding Stuffs. Horizontal Method for the Detection of Salmonella spp. Annex D: Detection of Salmonella spp. in Animal Faeces and in Samples from the Primary Production Stage. Geneve, Switzerland.

Keteran K, Brown J, Shotts EB Jr.: Salmonella in the mesenteric lymph nodes of healthy sows and hogs. Am J Vet Research 1982;43:706-707.
Mainar-Jaime RC, Andrés S, Vico JP, San Román B, Garrido V, Grilló MJ. Sensitivity of the ISO 6579:2002/Amd 1:2007 standard method for detection of Salmonella spp. on mesenteric lymph nodes from slaughter pigs. J Clin Microbiol 2013; 51:89-94.

MAPA Ministry of Agriculture Fisheries and Food. The pork industry in figures: Main economic indicators in 2017. Deputy general manager of livestock producers 2018. Available at: https://www.mapa.gob.es/es/ganaderia/estadisticas/indicadores economicossectorporcinoano2018_tcm30-379728.pdf, accessed November 27, 2019.

Merle R, Kosters S, May T, Portsch U, Blaha T, Kreienbrock L. Serological Salmonella monitoring in German pig herds: Results of the years 2003-2008. Prev Vet Med 2011;99:229233.

Meroc E, Strubbe M, Vangroenweghe F, Czaplicki G, Vermeersch K, Hooyberghs J. Van der Stede Y. Evaluation of the Salmonella surveillance program in Belgian pig farms. Prev Vet Med 2012;105:309-314.

Missotten JA, Michiels J, Degroote J, De Smet S. Fermented liquid feed for pigs: An ancient technique for the future. J Anim Sci Biotechnol 2015;6:4.

Mousing J, Jensen PT, Halgaard C, Bager F, Feld N, Nielsen B, Nielsen JP, Bech-Nielsen S. Nation-wide Salmonella enterica surveillance and control in Danish slaughter swine herds. Prev Vet Med 1997;29:247-261.

San Román B, Garrido V, Sánchez S, Martínez-Ballesteros I, Garaizar J, Mainar-Jaime RC, Migura-García L, Grilló MJ. Relationship between Salmonella infection, shedding and serology in fattening pigs in moderate prevalence areas. Zoonoses Public Health 2018;65:481-489.

Scherer K, Szabo I, Rosler U, Appel B, Hensel A, Nockler K. Time course of infection with Salmonella Typhimurium and its influence on fecal shedding, distribution in inner organs, and antibody response in fattening pigs. J Food Prot 2008;71: 699-705.

Van Winsen RL, Lipman LJ, Biesterveld S, Urlings BA, Snijders JM, Van Knapen F. Mechanism of Salmonella reduction in fermented pig feed. J Sci Food Agric 2000;81:342-346.

Van Winsen RL, Van Nes A, Keuzenkamp D,. Urlings HA, Lipman LJ, Biesterveld S, Snijders JM, Verheijden JH, Van Knapen F. Monitoring of transmission of Salmonella enterica serovars in pigs using bacteriological and serological detection methods. Vet Microbiol 2001;80:267-274.

Vico JP, Engel B, Buist WG, Mainar-Jaime RC. Evaluation of three commercial enzyme-linked immunosorbent assays for the detection of antibodies against Salmonella spp. in meat juice from finishing pigs in Spain. Zoonoses Public Health 2010;57(Suppl. 1):107-114.

Vico JP, Mainar-Jaime RC. The use of meat juice or blood serum for the diagnosis of Salmonella infection in pigs and its possible implications on Salmonella control programs. J Vet Diagn Invest 2011;23:528-531.

Address correspondence to: María-Jesús Grilló, PhD Instituto de Agrobiotecnología (CSIC-Gobierno de Navarra)

Grupo de Sanidad Animal

Avenida de Pamplona, 123 Mutilva, Navarra 31192

Spain

E-mail:mj.grillo@csic.es 